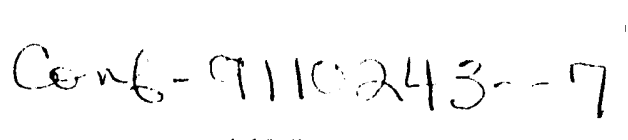

BNL -46751

\author{
5th ICFA Advanced Beam Dynamics Workshop \\ Corpus Christi, Texas \\ October 3-8, 1991
}

\title{
Exact Analysis to any Order of the Linear Coupling Problem in the Thin Lens Model
}

\author{
Alessandro G. Ruggiero \\ Brookhaven National Laboratory,* Upton, INY 11973 \\ BNL --46751 \\ DE92 005005
}

\begin{abstract}
In this report we attempt the exact solution of the motion of a charged particle in a circular accelerator under the effects of skew quadrupcle errors. We adopt the model of error distributions, lumped in locations with cero extension. This thin-lens approximation provides an analytical insight to the problem to any order. The total solution is expressed in terms of driving terms which are actually correlation factors to several order. An application follows on the calculation and correction of tune-splitting and on the estimate of the role the higher-order terms play in the correction method.
\end{abstract}

\subsection{Introduction}

Despite the very simple form of the equations of motion and the knuwledge that the motion is exactly integrable, the complete solution of the linear coupling problem in a practical form remains an outstanding issue. The complication arises from the fact that the solution is to be described in a four-dimensional phase space which is difficult to perceive. Several approaches and general analysis have been proposed in the past. ${ }^{1,2,3}$ In our opinion, nevertheless the methods used are too abstract; aside from the formulation of some general and important theorems, they do not really provide much irsight into the problem and can be hardly useful for designing correction schemes of the coupling effects.

In the approximation that the skew quadrupole errors causing the coupling are small, it is possible to apply the perturbation method ${ }^{3}$ to the solution of the problem. But also this may not be sufficient; for instance in the case of storage rings made of superconducting magnets, these are known to be susceptible to rather large skew quadrupole errors. In this case, a more complete and accurate analysis is required since high-order effects are expected to play important role.

Recently, it has become common practice to simulate the effect of linear coupling in particle tracking on computers. The errors are usually taken as lumped in location of zero length. The effects are then determined either by studying directly the particle motion or by solving for the eigenvalues of the resulting $4 \times 4$ transfer matrix. We believe that the thin-lens model of the errors is indeed adequate and formally correct. We have taken this model as our starting point for the analysis that follows since, as we shall $s z e$, it is indeed suitable for an exact analytical treatment with essentially no approximation involved and with an accuracy well seyond of that one can obtain with the perturbation method. Inc sed, s we shall see, we have been capable to determin s the importanc: of the higher order effects and the role they play in a particular correction scheme.

* Work performed under the auspices of the U.S. Department of Energy. 


\subsection{Thin-Lens Model of the Errors}

Let us take a distribution of skew quadrupole errors around a circular accelerator or storage ring. Each error will be approximated as a thin lens described by the strength parameter

$$
\mathrm{g}_{\mathrm{i}}=\left(\frac{\mathrm{B}^{\prime} \ell}{\mathrm{B} \rho}\right)_{\mathrm{i}}
$$

where $\mathrm{i}=1,2, \ldots \mathrm{N}$ and $\mathrm{N}$ is the total number of errors in the ring. The strength parameter is the gradient error $\mathrm{B}^{\prime}$ multiplied by the length $\ell$ of the error source and divided by the magnetic rigidity $\mathrm{B} \rho$ of the charged particle being accelerated or circulating in the storage ring. If $s$ is the curvilinear coordinate along the reference orbit, we shall take a point of observation as a starting point at $s=s_{0}$ and assume that the $i$-th skew quadrupole error is lumped at the location $s=s_{i}$. Our first goal is the derivation of the one-turn $4 \times 4$ transfer matrix $M\left(s_{0}\right)$ which describes the motion of a charged particle in linear approximation moving from $s=s_{0}$ to $s=s_{0}+L$ where $L$ is the total circumference of the reference closed orbit. For this purpose we shall refer to Fig. 1 which shows the location of the skew quadrupole errors and of partial transfer matrices. We shall define with $M_{i}$ the $4 \times 4$ matrix which takes the motion from $s=s_{i-1}$ to $s=s_{i}$, that is between two errors placed next to each other, and with $Q_{i}$ the $4 \times 4$ matrix associated to the $i$-th error and which describes its effect. In particular $M_{1}$ takes the motion from the starting point $s=s_{0}$ to the location of the first error $s=s_{1}$, and $M_{N+1}$ takes the motion from the location of the last error at $s=s_{N}$ back to the origin at $s=s_{0}$. This model, which is based on the short-length and on the discontinuous nature of the errors, is a fair and realistic approximation of a particle accelerator which avoids the introduction of continuous distributions of errors. After all, this model is commonly used in computer particle tracking with programs employing the kick method.

The total transier matrix $M$ from $s=s_{0}$ to $s=s_{0}+L$ is easily put together as follows

$$
M=M_{N+1}\left(Q_{N} M_{N}\right) \cdots\left(Q_{i+1} M_{i+1}\right)\left(Q_{i} M_{i}\right) \cdots\left(Q_{2} M_{2}\right)\left(Q_{1} M_{1}\right)
$$

There is an easily identifiable pattern which we have emphasized by grouping elements within brackets. It is a chain of similar factors $\mathrm{Q}_{\mathrm{i}} \mathrm{M}_{\mathrm{i}}$ made by the product of a kick matrix and the transfer matrix from the preceeding error.

Between errors the motion is decoupled and

$$
\mathrm{M}_{\mathrm{i}}=\left(\begin{array}{cc}
M_{H i} & \emptyset \\
\emptyset & M_{V i}
\end{array}\right)
$$

where $\mathrm{M}_{\mathrm{Hi}}$ is the ordinary horizontal and $\mathrm{MVi}_{\mathrm{i}}$ vertical $2 \times 2$ matrix from $s=s_{i-1}$ to $s=s_{i}$ and $\emptyset$ is the null $2 \times 2$ matrix

$$
\emptyset=\left(\begin{array}{ll}
0 & 0 \\
0 & 0
\end{array}\right)
$$

We also define the $2 \times 2$ unit matrix

$$
1=\left(\begin{array}{ll}
1 & 0 \\
0 & 1
\end{array}\right)
$$

The $4 \times 4$ matrix associated to the $\mathrm{i}$-th error is

$$
\mathrm{Q}_{\mathrm{i}}=\left(\begin{array}{cc}
1 & G_{\mathrm{i}} \\
G_{\mathrm{i}} & 1
\end{array}\right)
$$




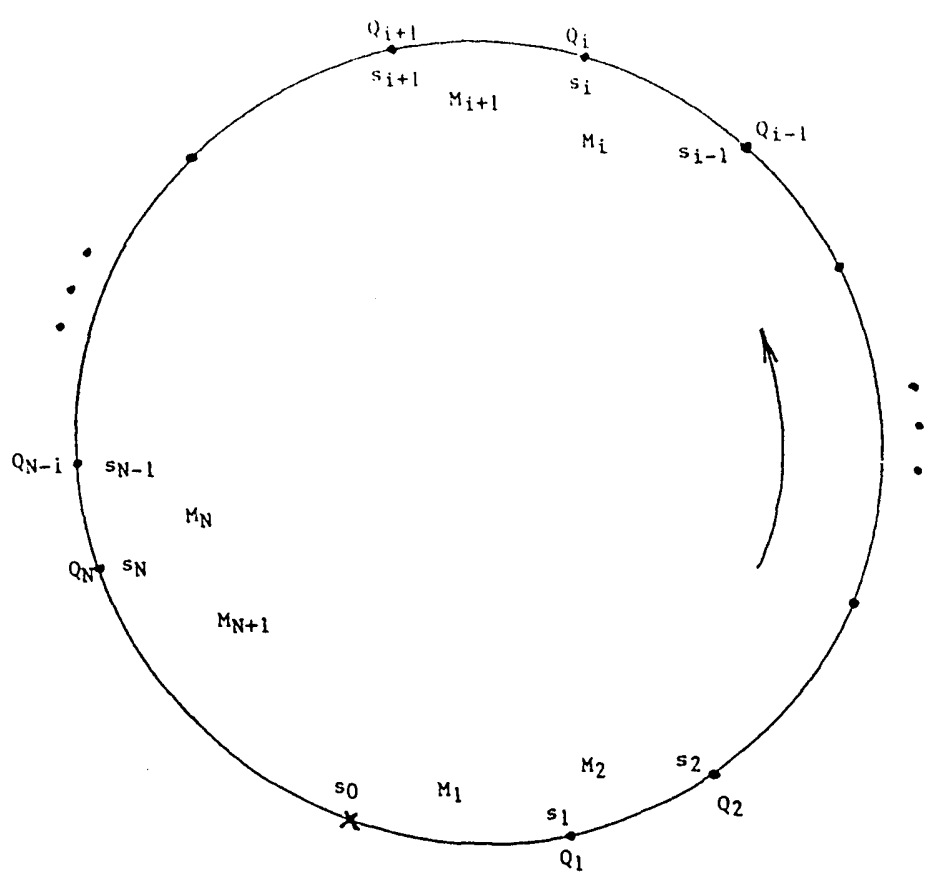

$M_{i}: 4 \times 4$ Transfer Matrix from $s=s_{i-1}$ to $s=s_{i}$.

$\mathrm{Q}_{\mathrm{i}}: 4 \times 4$ Transfer Matrix associated to i-th error. $\mathrm{s}_{\mathrm{i}}$ : location of $\mathrm{i}$-th error.

Figure 1: Distribution of Errors and Local Transfer Matrices.

where

$$
\mathrm{G}_{\mathrm{i}}=\left(\begin{array}{cc}
0 & 0 \\
g_{i} & 0
\end{array}\right)=\mathrm{g}_{\mathrm{i}} \mathrm{U}
$$

and

$$
\mathrm{U}=\left(\begin{array}{ll}
0 & 0 \\
1 & 0
\end{array}\right)
$$

It is easily seen that

$$
Q_{i}=\left(\begin{array}{ll}
1 & \emptyset \\
\emptyset & 1
\end{array}\right)+g_{i}\left(\begin{array}{cc}
0 & U \\
U & 0
\end{array}\right)
$$

so that finally

$$
\begin{aligned}
Q_{i} M_{i} & =M_{i}+g_{i}\left(\begin{array}{cc}
0 & U M_{V_{i}} \\
U M_{H_{i}} & \emptyset
\end{array}\right) \\
& =M_{i}+g_{i} T_{i}
\end{aligned}
$$

which shows a factorization with respect to the strength parameter.

By performing the required chain of multiplication to obtain $\mathrm{M}$, one derives a polynomial of the $\mathrm{N}$-th order in the errors $g_{i}, i=1,2, \ldots, N$, where the coeffcients are also $4 \times 4$ matrices. The problem is thus completely defined since we have already the explicit form of the $2 \times 2$ transfer matrices $M_{H i}$ and $M_{V_{i}}$ which are needed to give $M_{i}$ according to Eq. (3) and which will be given in the next section. Moreover, though $\mathrm{M}_{\mathrm{Hi}}$ and $\mathrm{M}_{\mathrm{Vi}}$ do not include skew quadrupole errors, they may include regular quadrupole errors, the magnitude and distribution of which is not necessary to specify. 


\subsection{Coordinate Normalization}

An inspection of Eq. (3) shows that only relatively few parameters enter the expression for the total transfer matrix $M$, namely: the strength parameter $g_{i}$ of the errors, the amplitude lattice functions $\beta_{H_{i}}$ and $\beta_{V_{i}}$ where the errors are located (H for horizontal and $V$ for vertical), their derivatives denoted with $\alpha_{H_{i}}$ and $\alpha_{V_{i}}$, and the betatron phase advances $\psi_{\mathrm{H}_{i}}$ and $\psi_{\mathrm{V}_{i}}$ at the location of the errors taken as difference from an arbitrarily chosen origin. As we have seen, the strength parameters can be factorized, but the values of the amplitude lattice functions and the phase advances appear together only in the transfer matrices $\mathbf{M}_{\mathbf{i}}$. A matrix transformation is known which allows also the factorization of the values of the amplitude lattice functions.

Introduce the matrix operator

$$
\mathrm{R}=\left(\begin{array}{cc}
R_{H} & \emptyset \\
\emptyset & R_{V}
\end{array}\right)
$$

where

$$
R_{H, V}=\left(\begin{array}{cc}
\frac{1}{\sqrt{\beta}} & 0 \\
\frac{\alpha}{\sqrt{\beta}} & \sqrt{\beta}
\end{array}\right)_{H_{1}, V}
$$

It is seen that Det $R_{H}=\operatorname{Det} R_{V}=\operatorname{Det} R=1$. The matrix $R^{-1}$ inverse to $R$ is

$$
\mathrm{R}^{-1}=\left(\begin{array}{cc}
R_{H}^{-1} & \emptyset \\
\emptyset & R_{V}^{-1}
\end{array}\right)
$$

where

$$
R_{\mathrm{H}, \mathrm{V}}^{-1}=\left(\begin{array}{cc}
\sqrt{\beta} & 0 \\
-\frac{\alpha}{\sqrt{\beta}} & \frac{1}{\sqrt{\beta}}
\end{array}\right)_{\mathrm{H}, \mathrm{V}}
$$

and

$$
\begin{gathered}
\mathrm{R}_{\mathrm{H}, \mathrm{V}}^{-1} \mathrm{R}_{\mathrm{H}, \mathrm{V}}=1 \\
\mathrm{R}^{-1} \mathrm{R}=\left(\begin{array}{ll}
1 & 0 \\
0 & 1
\end{array}\right)
\end{gathered}
$$

The operator $\mathrm{R}$ transforms the total transfer matrix $\mathrm{M}$ to $\mathcal{M}$ and allows the factorization of the values of the amplitude lattice functions,

$$
\mathcal{M}=\mathrm{R}_{0} \mathrm{M} \mathrm{R}_{\mathrm{o}}^{-1}
$$

where $R_{0}$ is the operator given by Eqs. (11 and 12) estimated at $s=s_{0}$. Let therefore $R_{i}$ (and its inverse $R_{i}^{-1}$ ) denote the same operator evaluated at the location $s=s_{i}$ of the $i$-th error; then, making use of the properties given by Eqs. (15 and 16), it is possible to combine Eqs. (2 and 17) as follows

$$
\begin{aligned}
\mathcal{M} & =R_{0} M_{N+1} R_{N}^{-1} R_{N} Q_{N} R_{N}^{-1} R_{N} M_{N} R_{N-1}^{-1} R_{N-1} Q_{N-1} \cdots \\
& \cdots R_{i+1}^{-1} R_{i+1} Q_{i+1} R_{i+1}^{-1} R_{i+1} M_{i+1} R_{i}^{-1} R_{i} Q_{i} R_{i}^{-1} R_{i} M_{i} \cdots \\
& \cdots R_{2}^{-1} R_{2} C_{2} R_{2}^{-1} R_{2} M_{2} R_{1}^{-1} R_{1} Q_{1} R_{1}^{-1} R_{1} M_{1} R_{0}^{-1}
\end{aligned}
$$

which has an obvious construction. The result is

$$
\begin{aligned}
\mathcal{M} & =\mathcal{M}_{N+1} \mathcal{Q}_{N} \mathcal{M}_{N} \mathcal{Q}_{N-1} \cdots \mathcal{Q}_{i+1} \mathcal{M}_{i+1} \mathcal{Q}_{i} \mathcal{M}_{i} \mathcal{Q}_{i-1} \cdots \\
& \cdots \mathcal{Q}_{2} \mathcal{M}_{2} \mathcal{Q}_{1} \mathcal{M}_{1}
\end{aligned}
$$


which is similar and equivalent to $M$ given by Eq. (2). It can be easily verified that

$$
\begin{aligned}
\mathcal{M}_{i} & =R_{i} M_{i} R_{i-1}^{-1} \\
& =\left(\begin{array}{cc}
\mathcal{M}_{H i} & \emptyset \\
\emptyset & \mathcal{M}_{V i}
\end{array}\right)
\end{aligned}
$$

equivalent to Eq. (3), and

$$
\mathcal{M}_{\mathrm{H}_{1} \mathrm{~V}_{\mathrm{i}}}=\left(\begin{array}{cc}
\cos \Delta_{H, V_{\mathrm{i}}} & \sin \Delta_{H, V_{\mathrm{i}}} \\
-\sin \Delta_{H, V_{\mathrm{i}}} & \cos \Delta_{H, V_{\mathrm{i}}}
\end{array}\right)
$$

where $\Delta_{\mathrm{H}, \mathrm{V}_{\mathrm{i}}}=\psi_{\mathrm{H}, \mathrm{V}_{\mathrm{i}}}-\psi_{\mathrm{H}, \mathrm{v}_{\mathrm{i}-1}}$ are the betatron phase advances from $\mathrm{s}=\mathrm{s}_{\mathrm{i}-1}$ to $\mathrm{s}=\mathrm{s}_{\mathrm{j}}$. The $2 \times 2$ matrices $\mathcal{M}_{\mathrm{H}_{\mathrm{i}}}$ and $\mathcal{M}_{\mathrm{V}_{\mathrm{i}}}$ represent rotations by the angles $-\Delta_{\mathrm{H}_{i}}$ and $-\Delta \mathrm{V}_{\mathrm{i}}$ respectively in the horizontal and vertical plane. It can also be seen that

$$
\begin{aligned}
\mathcal{Q}_{i} & =R_{i} Q_{i} R_{i}^{-1} \\
& =\left(\begin{array}{ll}
1 & 0 \\
0 & 1
\end{array}\right)+q_{i}\left(\begin{array}{ll}
0 & U \\
U & \emptyset
\end{array}\right)
\end{aligned}
$$

and

$$
\mathrm{q}_{\mathrm{i}}=\mathrm{g}_{\mathrm{i}} \sqrt{\beta_{\mathrm{H}_{\mathrm{i}}} \beta_{\mathrm{V}_{\mathrm{i}}}}
$$

is the strength parameter in normalized form. There is a very close analogy between Eq. (12) and Eq. (9), which are actually identical except for the replacement of $g_{i}$ with $q_{i}$.

The transformation given by Eq. (17) simplifies considerably the notation of the problem. It has the property of transforming trajectories in the betatron phase spaces that have elliptical shape to perfect circles. In particular the dependence on the derivatives $\alpha_{\mathrm{H}}$ and $\alpha_{\mathrm{V}}$ disappear explicitly. In the following, our goal is the study of the properties of the normalized matrix $\mathcal{M}$; when these are known, we can obtain the properties of the original matrix $M$ with the antitransformation

$$
M=R_{0}^{-1} \mathcal{M} R_{0}
$$

Our starting point is thus given by Eq. (19) where $\mathcal{M}_{i}$ is given by Eq. (20), which we have seen performs simple rotations and depends only on the betatron phase advances, and $\mathcal{Q}_{i}$ is the kick due to the error given by Eq. (22) in a factorized form which depends only on the normalized strength parameter $\mathrm{q}_{i}$ of the error given by Eq. (23).

Similarly to Eq. (10) also here we obtain

$$
\mathcal{Q}_{\mathrm{i}} \mathcal{M}_{\mathrm{i}}=\mathcal{M}_{\mathrm{i}}+\mathrm{q}_{\mathrm{i}} \tau_{\mathrm{i}}
$$

where

$$
\begin{aligned}
\tau_{i} & =\left(\begin{array}{cc}
0 & U \\
U & \emptyset
\end{array}\right) \mathcal{M}_{i} \\
& =\left(\begin{array}{cc}
0 & U \mathcal{M}_{V_{i}} \\
U \mathcal{M}_{H_{i}} & \emptyset
\end{array}\right)
\end{aligned}
$$




\subsection{The Polynominal Expansion}

After inserting Eq. (25) in the chain of products at the right side of Eq. (19), and having performed all the matrix multiplications, one obtains a polynominal which we can write as

$$
\mathcal{M}=\sum_{k=0}^{N} \mathcal{M}^{(k)}
$$

where $\mathcal{M}^{(k)}$ is $k$-th order term of the polynominal expansion. In particular it is seen that

$$
\begin{aligned}
\mathcal{M}^{(0)} & =\mathcal{M}_{N-1} \mathcal{M}_{N} \cdots \mathcal{M}_{i+1} \mathcal{M}_{i} \mathcal{M}_{i-1} \cdots \mathcal{M}_{2} \mathcal{M}_{1} \\
& =\mathcal{M}_{0}\left(s_{0}\right)=\mathcal{M}_{0}
\end{aligned}
$$

is the regular $4 \times 4$ transfer matrix which describes the motion in the storage ring in the absence of skew quadrupole errors (but including eventually regular quadrupole errors). Similarly

$$
\mathcal{M}^{(1)}=\sum_{i=1}^{N} q_{i} \mathcal{M}_{N+1} \mathcal{M}_{N} \cdots \mathcal{M}_{i+1} \mathcal{T}_{i} \mathcal{M}_{i-1} \cdots \mathcal{M}_{2} \mathcal{M}_{1}
$$

is the first-order term of the polynominal where $\mathcal{T}_{i}$ has been defined in Eq. (26), that is

$$
\tau_{\mathrm{i}}=\left(\begin{array}{cc}
0 & U \\
U & 0
\end{array}\right) \mathcal{M}_{\mathrm{i}}=\mathbf{U} \mathcal{M}_{\mathrm{i}}
$$

The second order term is

$$
\begin{gathered}
\mathcal{M}^{(2)}=\sum_{i=1}^{N-1} \sum_{j>i}^{N} q_{i} q_{j} \mathcal{M}_{N+1} \mathcal{M}_{N} \cdots \mathcal{M}_{j+1} \mathcal{T}_{j} \mathcal{M}_{j-1} \cdots \\
\ldots \mathcal{M}_{i+1} \mathcal{T}_{i} \mathcal{M}_{i-1} \cdots \mathcal{M}_{2} \mathcal{M}_{1}
\end{gathered}
$$

and so on; it is obvious how to construct the $k$-th order term $\mathcal{M}^{(k)}$. Finally, for instance,

$$
\begin{aligned}
\mathcal{M}^{(N-1)}= & \sum_{i=1}^{N}\left(q_{N} q_{N-1} \cdots q_{i+1} q_{i-1} \cdots q_{2} q_{1}\right) \mathcal{M}_{N+1} \mathcal{T}_{N} \mathcal{T}_{N-1} \cdots \\
& \cdots \mathcal{T}_{i+1} \mathcal{M}_{i} \mathcal{T}_{i-1} \cdots \mathcal{T}_{2} \mathcal{T}_{1}
\end{aligned}
$$

and

$$
\begin{aligned}
\mathcal{M}^{(N)}= & \left(q_{N} q_{N-1} \cdots q_{i+1} q_{i} q_{i-1} \cdots q_{2} q_{1}\right) \mathcal{M}_{N+1} \mathcal{T}_{N} \mathcal{T}_{N-1} \cdots \\
& \cdots \mathcal{T}_{i+1} \mathcal{T}_{i} \mathcal{T}_{i-1} \cdots \mathcal{T}_{2} \mathcal{T}_{1}
\end{aligned}
$$

Taking into account Eq. (30), after carrying obvious matrix multiplications, we have quite generally for the $\mathrm{k}$-th order term

$$
\begin{aligned}
\mathcal{M}^{(k)}= & \sum_{r_{1}=1} \sum_{r_{2}>r_{1}} \cdots \sum_{r_{k}>r_{k-1}}\left(q_{r_{1}} \cdots q_{r_{k}}\right) \mathcal{M}_{r_{k} 0} \mathrm{UM}_{r_{k-1} r_{k}} \cdots \\
& \cdots \mathcal{M}_{r_{1} r_{2}} \mathrm{UM}_{0 r_{1}}
\end{aligned}
$$

where $\mathcal{M}_{\mathrm{r}_{\mathrm{r}} \mathrm{j}}$ is the $4 \times 4$ transfer matrix from the location $\mathrm{s}=\mathrm{s}_{\mathrm{ri}}$ to the location $\mathrm{s}=\mathrm{s}_{\mathrm{rj}}$ without skew quadrupole errors in between.

$$
\mathcal{M}_{r_{i} r_{j}}=\left(\begin{array}{cc}
\mathcal{M}_{H_{r_{i} r_{j}}} & \emptyset \\
\emptyset & \mathcal{M}_{V_{r_{1}, j}}
\end{array}\right)
$$


where

$$
\mathcal{M}_{\mathrm{H}, V_{r_{i} r_{j}}}=\left(\begin{array}{cc}
\cos \Delta_{H, V_{r_{i} r_{j}}} & \sin \Delta_{H, V_{r_{i} r_{j}}} \\
-\sin \Delta_{H, V_{r_{i} r_{j}}} & \cos \Delta_{H, V_{r_{i} r_{j}}}
\end{array}\right)
$$

and

$$
\Delta_{\mathrm{H}, \mathrm{V}_{\mathrm{r}_{\mathrm{i}} \mathrm{r}_{\mathrm{j}}}}=\psi_{\mathrm{H}, \mathrm{V}_{\mathrm{r}_{\mathrm{j}}}}-\psi_{\mathrm{H}, \mathrm{V}_{\mathbf{r}_{\mathrm{i}}}}
$$

The $k$-th order term of the polynominal expansion, given by Eq. (34), includes $k$ sums with $k$ different indices $r_{1}, r_{2}, \ldots, r_{k}$. The sum with the index $r_{k}$ ranges from $r_{k-1}+1$ to $N$; the sum with index $r_{k-1}$ ranges from $r_{k-2}+1$ to $N-1$, and so forth; at last, the summation with index $r_{1}$ ranges from 1 to $\mathrm{N}-\mathrm{k}+1$. In each sum there is always a scalar factor represented by the product of $k$ strength parameters of the errors $\left(q_{r_{1}} \cdots q_{r_{k}}\right)$ : thus the label "k-th order" given to $\mathcal{M}^{(k)}$ of $\mathrm{Eq}$. (34). It is to be observed that the parameter strengths appearing in the scalar factor are all different from each other. Finally, it appears a product of regular transfer matrices $\mathcal{M}_{\mathrm{r}_{\mathrm{r}} \text {; }}$ from one location to the other of the selected skew quadrupole errors according to the summation rule specified. These transfer matrices are separated from each other by the insertion of the $4 \times 4$ matrix $U$ defined in Eq. (30), and which represent the effects of the kicks due to the errors.

A close inspection of the form of the $\mathrm{k}$-th order term of the polynominal expansion given by Eqs. (27 and 34) and the understanding of its construction allow, as we shall see next, tr: application of simple and straightforth operation that only at first sight may look complicated and cumbersome.

Since it is reasonable to expect that the strength parameters of the errors $q_{i}$ defined by Eq. (23) are small, one may be tempted to apply perturbation methods to estimate their effects on the particle motion. For instance, once obtained the polynominal expansion given by Eqs. (27 and 34), it could be possible to retain only the first-order and second-order term $(k=1$ and 2$)$ and truncate the expansion by neglecting the higher-order terms $(k>2)$. This approach is invalid because it does not preserve the symplectic properties of the total transfer matrix $\mathcal{M}$, which are also implicitly assumed in the unperturbed matrix $\mathcal{M}_{0}$ and in each of the partial transfer matrices $\mathcal{M}_{\mathrm{r}_{i} \mathrm{r}_{j}}$. It is well known that the product of symplectic matrices is also symplectic; but that is not true if at least one of the matrices in the product is not symplectic. This is the case for each of the term $\mathcal{M}^{(k)}$ in the polynominal expansion with the exclusion of $\mathcal{M}^{(0)}=\mathcal{M}_{0}$. From Eq. (34) which gives the explicit form of $\mathcal{M}^{(k)}$, it is to be noticed the inclusion of the matrix $U$ which is not symplectic. Thus each term $\mathcal{M}^{(k)}$ is not a symplectic operator, though their sum, including $\mathrm{k}=0$, gives a symplectic operator. A truncation of the expansion given by Eq. (27) to an order $r_{k}<N$ is therefore not symplectic. To avoid this problem, it is our intention to retain all the terms of the expansion and calculate them explicitly from Eq. (34).

\subsection{Complex Number Notation}

It is more convenient to describe the $4 \times 4$ total transfer matrix $\mathcal{M}$ in terms of four $2 \times 2$ matrices as follows

$$
\begin{aligned}
\mathcal{M} & =\left(\begin{array}{ll}
A & n \\
m & B
\end{array}\right) \\
& =\sum_{k=0}^{N}\left(\begin{array}{ll}
A^{(k)} & n^{(k)} \\
m^{(k)} & B^{(k)}
\end{array}\right)
\end{aligned}
$$

Obviously $\mathrm{n}^{(0)}=\mathrm{m}^{(0)}=0$ and $\mathrm{A}^{(0)}, \mathrm{B}^{(0)}$ reduce respectively to the matrices $\mathcal{M}_{\mathrm{H}}, \mathcal{M}_{\mathrm{V}}$ which describe the one- turn transformation respectively in the horizontal and vertical plane in the 
absence of skew quadrupole errors but eventually with the inclusion of regular quadrupole errors, since these do not affect the off-diagonal $2 \times 2$ matrices $\mathrm{n}$ and $\mathrm{m}$.

The transfer matrix $\mathrm{M}$ given by $\mathrm{Eq}$. (2) operates on a four-dimensional vector $\mathrm{Z}$ which describes the status of motion of a charged partial at the location $s=s_{0}$

$$
\mathrm{Z}=\left(\begin{array}{l}
X \\
Y
\end{array}\right)
$$

where

$$
\mathrm{X}=\left(\begin{array}{c}
x \\
x^{\prime}
\end{array}\right) \quad \text { and } \mathrm{Y}=\left(\begin{array}{c}
y \\
y^{\prime}
\end{array}\right)
$$

and $x, x^{\prime}, y, y^{\prime}$ represent the usual particle coordinates and angles. On the other hand the matrix $\mathcal{M}$ given by $\mathrm{Eq}$. (19) will operate on the vector

$$
\mathcal{Z}=\mathrm{R}_{0} \mathrm{Z}
$$

which represents the position of the particle in the four-dimensional phase space in the normalized form. Similarly to Eqs. (39) and (40) we have

$$
\mathcal{Z}=\left(\begin{array}{l}
\mathcal{X} \\
\mathcal{Y}
\end{array}\right)
$$

where

$$
\mathcal{X}=\left(\begin{array}{c}
u \\
u^{\prime}
\end{array}\right) \text { and } \mathcal{Y}=\left(\begin{array}{c}
v \\
v^{\prime}
\end{array}\right)
$$

and $u, u^{\prime}, v, v^{\prime}$ represe.t whe particle coordinates and angles in the normalized form. It is seen that $A^{(k)}$ and $m^{(k)}$ are $2 \times 2$ matrices which always operate on the two-dimensional vector $\mathcal{X}$, whereas $B^{(k)}$ and $n^{(k)}$ are $2 \times 2$ matrices acting only on the two-dimensional vector $\mathcal{Y}$.

Instead of the two-dimensional vectors and matrices it is also possible to use an equivalent complex number notation. For instance the vector $\mathcal{X}$ can be replaced by

$$
\mathcal{X}=\rho \mathrm{e}^{\mathrm{i} \theta}
$$

with amplitude

$$
\rho=\sqrt{\mathrm{u}^{2}+\mathrm{u}^{\prime 2}}
$$

and phase

$$
\theta=\operatorname{arctg} \mathrm{u}^{\prime} / \mathrm{u}
$$

Matrices $\mathrm{n}, \mathrm{m}, \mathrm{A}$ and $\mathrm{B}$ then become operators acting on amplitudes and phases. In particular, matrices $\mathcal{M}_{r_{i} r_{j}}$ appearing in Eq. (34) have the property, as we have seen, of rotations; they leave the amplitude unchanged and shift the phase $\theta$ in the clockwise direction by an angle $\Delta_{\mathrm{H}_{\mathrm{r}_{\mathrm{i}} \mathrm{r}_{\mathrm{j}}}}$ in the horizontal plane and $\Delta \mathrm{V}_{\mathrm{r}_{\mathrm{i}} \mathrm{r}_{\mathrm{j}}}$ in the vertical plane. The matrix operator $U$ has a more interesting property

$$
\begin{aligned}
U \mathcal{X} & =\left(\begin{array}{ll}
0 & 0 \\
1 & 0
\end{array}\right)\left(\begin{array}{c}
\rho \cos \theta \\
\rho \sin \theta
\end{array}\right)=\left(\begin{array}{c}
0 \\
\rho \cos \theta
\end{array}\right) \\
& =i \rho \cos \theta=\frac{i}{2} \rho\left(e^{i \theta}+e^{-i \theta}\right) \\
& =\frac{i}{2}\left(\mathcal{X}+\mathcal{X}^{*}\right) \\
& =\frac{i}{2}(1+C) \mathcal{X}
\end{aligned}
$$


where $\mathcal{X}^{*}$ is the complex conjugate of $\mathcal{X}$ and $\mathrm{C}$ is the operator that applied to $\mathcal{X}$ changes $\mathcal{X}$ to its complex conjugate. In matrix notation

$$
C=\left(\begin{array}{rr}
1 & 0 \\
0 & -1
\end{array}\right)
$$

A similar derivation is obtained also for $U \mathcal{Y}$.

\subsection{Derivation of Driving Terms to All Orders}

By comparing Eq. (38) with Eq. (34) we obtain the following formal construction of the $2 \times ?$ transfer matrix $\mathbf{n}^{(\mathrm{k})}$

$$
\begin{aligned}
& n^{(k)}=\sum_{r_{2}=1} \sum_{r_{2}>r_{1}} \cdots \sum_{r_{k}>r_{k-1}}\left(q_{1} \cdots q_{r_{k}}\right) \times \\
& \times \mathcal{M}_{H} U \mathcal{M}_{V} U \mathcal{M}_{H} \cdots \mathcal{M}_{V} U \mathcal{M}_{H} U \mathcal{M}_{V} \\
& \begin{array}{lllll}
r_{k} & r_{k-1} & \cdots & r_{2} & r_{1}
\end{array}
\end{aligned}
$$

which is valid for $\mathrm{k}$ odd; if $\mathrm{k}$ is even then $\mathrm{n}^{(\mathrm{k})}=0$. In the expression above, $\mathcal{M}_{\mathrm{H}, \mathrm{V}}$ are the $2 \times 2$ partial transfer matrices (rotation operators) as defined by Eqs. (35-37) and $U$ is the matrix operator defined by Eq. (8) and Eq. (47). There are k summations involved as already

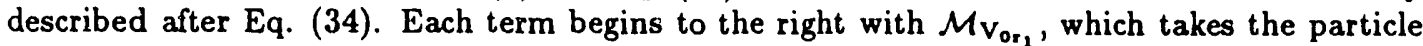
from the origin at $s=s_{0}$ to the location $s=s_{\mathrm{r}_{1}}$ of the selected error by the summation rules: at this location the operator $U$ is applied and it is followed now by $\mathcal{M}_{\mathrm{H}_{\mathrm{r}_{1}, r_{2}}}$ which takes the particle from $s=s_{r_{1}}$ to the second selected errors locations at $s=s_{r_{2}}$, to be followed also by the operator $U$ again. This procedure repeats until the particle is brought from $s=s_{r_{k}}$, the location of the last of the selected errors, back to the origin by $\mathcal{M}_{\mathbf{H}_{\mathbf{r}_{\mathbf{k}}} \mathbf{O}}$. It takes some careful study, but then the explicit construction of $n^{(k)}$ becomes obvious.

An expression very similar to Eq. (49) is also valid for $A^{(k)}$ with the exception that the order $k$ is now even; if $k$ is odd then $A^{(k)}=0$. Moreover all the terms in the summation begin to the right with $\mathcal{M}_{\mathrm{H}_{0} r_{1}}$ this time. Finally the $2 \times 2$ matrices $\mathrm{m}^{(\mathrm{k})}$ and $\mathrm{B}^{(\mathrm{k})}$ can be obtained from $n^{(k)}$ and $A^{(k)}$ respectively by simply replacing $\mathcal{M}_{H}$ in Eq. (49) with $\mathcal{M V}_{V}$ and vice versa. Thus by deriving more explicit expressions for $n^{(k)}$ from Eq. (49), one learns how to obtain equivalent expressions also for $\mathrm{m}^{(\mathrm{k})}, \mathrm{A}^{(\mathrm{k})}$ and $\mathrm{B}^{(\mathrm{k})}$.

With the insertion of Eq. (47) in a. (49) we obtain

$$
\begin{aligned}
& n^{(k)}=\left(\frac{i}{2}\right)^{k} \sum_{r_{1}=1} \sum_{r_{2}>r_{1}} \cdots \sum_{r_{k}>r_{k-1}}\left(q_{1} \cdots q_{k}\right) \times \\
& \times \mathcal{M}_{H}(1+C) \mathcal{M}_{V}(1+C) \mathcal{M}_{H} \cdots \mathcal{M}_{V}(1+C) \mathcal{M}_{H}(1+C) \mathcal{M}_{V} \\
& \begin{array}{lllll}
r_{k} & r_{k-1} & \cdots & r_{2} & r_{1}
\end{array}
\end{aligned}
$$

that is

$$
\begin{array}{r}
n^{(k)}=\left(\frac{i}{2}\right)^{k} e^{-i \mu_{H}}\left\{\left(J_{1}^{(k)}+J_{2}^{(k)}+\cdots+J_{h_{k}}^{(k)}\right)+\right. \\
\left.+\left(K_{1}^{(k)}+K_{2}^{(k)}+\cdots+K_{h_{k}}^{(k)}\right) C\right\}
\end{array}
$$

where $\mu_{\mathrm{H}, \mathrm{V}}=2 \pi \nu_{\mathrm{H}, \mathrm{V}}$ is the total phase advance per revolution. 
At the right side of Eq. (51) there are a total of

$$
2 h_{k}=2^{k}
$$

driving terms of the $k$-th order, divided in two equal groups: those of difference mode $\mathrm{J}_{\ell}^{(\mathrm{k})}$ and those of sum mode $\mathrm{K}_{\ell}^{(\mathrm{k})}$ where $\ell=1,2, \ldots, \mathrm{h}_{\mathrm{k}}$. In the following it will be convenient to define the phase difference

$$
\delta_{\mathrm{r}}=\left(\psi_{\mathrm{H}}-\psi_{\mathrm{V}}\right)_{\mathrm{r}}
$$

and sum

$$
\sigma_{\mathrm{r}}=\left(\psi_{\mathrm{H}}+\psi_{\mathrm{V}}\right)_{\mathrm{r}}
$$

at the location $s=s_{r}$. It can be shown that

$$
J_{\ell}^{(k)}=\sum_{r_{1}=1} \sum_{r_{2}>r_{1}} \cdots \sum_{r_{k}>r_{k-1}}\left(q_{1} \cdots q_{r_{k}}\right) e^{i \eta_{l}^{(k)}}
$$

and

$$
K_{\ell}^{(k)}=\sum_{r_{1}=1} \sum_{r_{2}>r_{1}} \cdots \sum_{r_{k}>r_{k-1}}\left(q_{1} \cdots q_{r_{k}}\right) e^{i} \zeta_{l}^{(k)}
$$

where $\eta_{l}^{(k)}$ and $\zeta_{l}^{(k)}$ are given by an algebraic combınation of phase differences and sums at different locations. For instance

$$
\begin{aligned}
& \eta_{1}^{(1)}=\delta_{r_{1}}=\left(\psi_{H}-\psi_{V}\right)_{r_{1}} \\
& \zeta_{1}^{(1)}=\sigma_{r_{1}}=\left(\psi_{H}+\psi_{V}\right)_{r_{1}}
\end{aligned}
$$

so that, in particular

$$
\begin{aligned}
J_{1}^{(1)} & =\sum_{r=1}^{N} q_{r} e^{i\left(\psi_{H}-\psi_{V}\right)_{r}} \\
& =J_{-}
\end{aligned}
$$

and

$$
\begin{aligned}
K_{1}^{(1)} & =\sum_{r=1}^{N} q_{r} e^{i\left(\psi_{H}+\psi_{V}\right)_{r}} \\
& =J_{+}
\end{aligned}
$$

In order to obtain the phase angles $\eta_{l}^{(k)}$ and $\zeta_{l}^{(k)}$ of the k-th order the following construction rules apply:

(i) Assume that the lower order $\eta_{\ell}^{(k-1)}$ and $\zeta_{\ell}^{(k-1)}$ phase angles are known;

(ii) shift the summation indices by one order upward, that is $\mathbf{r}_{\mathbf{j}} \rightarrow \mathbf{r}_{\mathbf{j}+1}$;

(iii) if $k$ is odd (that is $n^{(k)}$ )

- the $\eta_{l}^{(\mathrm{k})}$ angles are obtained by adding $+\delta_{\mathrm{r}_{1}}$ to each $\eta_{l}^{(\mathrm{k}-1)}$ and $-\sigma_{\mathrm{r}_{1}}$ to each $\zeta_{l}^{(\mathrm{k}-1)}$,

- the $\zeta_{\ell}^{(k)}$ angles are obtained by adding $-\delta_{r_{1}}$ to each $\zeta_{\ell}^{(k-1)}$ and $+\sigma_{r_{1}}$ to each $\eta_{\ell}^{(k-1)}$;

(iv) if $k$ is even (that is $A^{(k)}$ )

- the $\eta_{l}^{(\mathrm{k})}$ angles are obtained by adding $-\delta_{r_{1}}$ to each $\eta_{l}^{(\mathrm{k}-1)}$ and $-\sigma_{\mathrm{r}_{1}}$ to each $\zeta_{l}^{(\mathrm{k}-1)}$,

- the $\zeta_{l}^{(k)}$ angles are obtained by adding $+\delta_{r_{1}}$ to each $\zeta_{l}^{(k-1)}$ and $+\sigma_{r_{1}}$ to each $\eta_{l}^{(k-1)}$. 
In particular,

$$
\mathrm{n}^{(1)}=\frac{\mathrm{i}}{2} \mathrm{e}^{-1 \mu_{H}}\left(\mathrm{~J}_{1}^{(1)}+\mathrm{K}_{1}^{(1)} \mathrm{C}\right)
$$

where $\mathrm{J}_{1}^{(1)}$ and $\mathrm{K}_{1}^{(1)}$ have already been defined by Eqs. (59 and 60). Also

$$
\begin{array}{r}
A^{(2)}=-\frac{1}{4} e^{-i \mu_{H}}\left[J_{1}^{(2)}+J_{2}^{(2)}+\right. \\
\left.+\left(K_{1}^{(2)}+K_{2}^{(2)}\right) C\right]
\end{array}
$$

where

$$
\begin{aligned}
J_{1}^{(2)} & =\sum_{r_{1}=1}^{N-1} \sum_{r_{2}>r_{1}}^{N} q_{r_{1}} q_{r_{2}} e^{i\left(\delta_{r_{2}}-\delta_{r_{1}}\right)} \\
J_{2}^{(2)} & =\sum_{r_{1}=1}^{N-1} \sum_{r_{2}>r_{1}}^{N} q_{r_{1}} q_{r_{2}} e^{i\left(\sigma_{r_{2}}-\sigma_{r_{1}}\right)} \\
K_{1}^{(2)} & =\sum_{r_{1}=1}^{N-1} \sum_{r_{2}>r_{1}}^{N} q_{r_{1}} q_{r_{2}} e^{i\left(\sigma_{r_{3}}+\delta_{r_{1}}\right)} \\
K_{2}^{(2)} & =\sum_{r_{1}=1}^{N-1} \sum_{r_{2}>r_{1}}^{N} q_{r_{1}} q_{r_{2}} e^{i\left(\delta_{r_{2}}+\sigma_{r_{2}}\right)}
\end{aligned}
$$

and

$$
\begin{aligned}
& n^{(3)}=-\frac{i}{8} e^{-i \mu_{H}}\left[J_{1}^{(3)}+J_{2}^{(3)}+J_{3}^{(3)}+J_{4}^{(3)}+\right. \\
& \left.+\left(K_{1}^{(3)}+K_{2}^{(3)}+K_{3}^{(3)}+K_{4}^{(3)}\right) C\right]
\end{aligned}
$$

where

$$
\begin{aligned}
J_{1}^{(3)} & =\sum_{r_{1}=1}^{N-2} \sum_{r_{2}>r_{1}}^{N-1} \sum_{r_{3}>r_{2}}^{N} q_{r_{1}} q_{r_{2}} q_{r_{3}} e^{i\left(\delta_{r_{3}}-\delta_{r_{2}}+\delta_{r_{1}}\right)} \\
J_{2}^{(3)} & =\sum_{r_{1}=1}^{N-2} \sum_{r_{2}>r_{1}}^{N-1} \sum_{r_{3}>r_{2}}^{N} q_{r_{1}} q_{r_{3}} q_{r_{3}} e^{i\left(\sigma_{r_{3}}-\sigma_{r_{2}}+\delta_{r_{1}}\right)} \\
J_{3}^{(3)} & =\sum_{r_{1}=1}^{N-2} \sum_{r_{2}>r_{1}}^{N-1} \sum_{r_{3}>r_{2}}^{N} q_{r_{1}} q_{r_{2}} q_{r_{3}} e^{i\left(\sigma_{r_{3}}+\delta_{r_{2}}-\sigma_{r_{1}}\right)} \\
J_{4}^{(3)} & =\sum_{r_{1}=1}^{N-2} \sum_{r_{2}>r_{1}}^{N-1} \sum_{r_{3}>r_{2}}^{N} q_{r_{1}} q_{r_{2}} q_{r_{3}} e^{i\left(\delta_{r_{3}}+\sigma_{r_{2}}-\sigma_{r_{1}}\right)} \\
K_{1}^{(3)} & =\sum_{r_{1}=1}^{N-2} \sum_{r_{2}>r_{1}}^{N-1} \sum_{r_{3}>r_{2}}^{N} q_{r_{1}} q_{r_{2}} q_{r_{3}} e^{i\left(\sigma_{r_{3}}+\delta_{r_{2}}-\delta_{r_{1}}\right)} \\
K_{2}^{(3)} & =\sum_{r_{1}=1}^{N-2} \sum_{r_{2}>r_{1}}^{N-1} \sum_{r_{3}>r_{2}}^{N} q_{r_{1}} q_{r_{2}} q_{r_{3}} e^{i\left(\delta_{r_{3}}+\sigma_{r_{2}}-\delta_{r_{1}}\right)}
\end{aligned}
$$




$$
\begin{aligned}
K_{3}^{(3)} & =\sum_{r_{1}=1}^{N-2} \sum_{r_{2}>r_{1}}^{N-1} \sum_{r_{3}>r_{2}}^{N} q_{r_{1}} q_{r_{2}} q_{r_{3}} e^{i\left(\delta_{r_{3}}-\delta_{r_{2}}+\sigma_{r_{1}}\right)} \\
K_{4}^{(3)} & =\sum_{r_{1}=1}^{N-2} \sum_{r_{2}>r_{1}}^{N-1} \sum_{r_{3}>r_{2}}^{N} q_{r_{1}} q_{r_{2}} q_{r_{3}} e^{i\left(\sigma_{r_{3}}-\sigma_{r_{2}}+\sigma_{r_{1}}\right)}
\end{aligned}
$$

and so on... Though the construction of the driving terms is by now obvious, nevertheless their bookkeeping and notation is complicated.

\subsection{Search of the Eigenvalues}

There are four eigenvalues $\lambda_{1}, \lambda_{2}, \lambda_{3}$ and $\lambda_{4}$ which are solution of the equation

$$
\mathcal{M} \mathcal{Z}=\lambda \mathcal{Z}
$$

The general discussion and solution of this equation can be found elsewhere. ${ }^{1}$ Here, for our purposes, we shall notice the following. Since $\mathcal{M}$ is a symplectic matrix, if $\lambda$ is an eigenvalue, the complex conjugate $\lambda^{*}$ is also an eigenvalue. Moreover if $\lambda$ is an eigenvalue then the inverse $\lambda^{-1}$ is also an eigenvalue. Thus the eigenvalues can be paired; for instance, we can define

$$
\begin{aligned}
& \Lambda_{1}=\lambda_{1}+1 / \lambda_{1}=2 \cos \mu_{1} \\
& \Lambda_{2}=\lambda_{2}+1 / \lambda_{2}=2 \cos \mu_{2}
\end{aligned}
$$

It can be proven that 1

$$
\begin{aligned}
& \cos \mu_{1}+\cos \mu_{2}=\frac{1}{2}(\operatorname{Tr} A+\operatorname{Tr} B) \\
& \cos \mu_{1}-\cos \mu_{2}=\sqrt{\frac{1}{4}(\operatorname{Tr} A-\operatorname{Tr} B)^{2}+\operatorname{Det}(n+\bar{m})}
\end{aligned}
$$

where $A, B, n$ and $m$ are the $2 \times 2$ matrices as they appear in $E q .(38)$, and $\bar{m}$ is the matrix symplectic conjugate to $\mathrm{m}$, (see ref. 1.). Also

$$
\begin{aligned}
4 \cos \mu_{1,2} & =(\operatorname{Tr} A+\operatorname{Tr} B)+ \\
& \pm \sqrt{(\operatorname{Tr} A-\operatorname{Tr} B)^{2}+4 \operatorname{Det}(n+\bar{m})}
\end{aligned}
$$

We notice that we are dealing with $2 \times 2$ matrices which are linear combinations of simple rotation operators and of the complex conjugate operator $C$. Thus, using also known properties of symplectic matrices, we have (see Appendix A and B)

$$
\begin{aligned}
& \operatorname{Det}(n+\bar{m})= \\
& =\sum_{\substack{k_{1}=1 \\
\text { odd }}}^{N} \sum_{\substack{k_{2}=1 \\
\text { odd }}}^{N} \sum_{\ell_{1}=1}^{h_{k_{1}}} \sum_{l_{2}=1}^{h_{k_{2}}}\left(\frac{i}{2}\right)^{k_{1}}\left(-\frac{i}{2}\right)^{k_{2}} \times \\
& \times \operatorname{Real}\left\{J_{\ell_{1}}^{\left(k_{1}\right)} J_{\ell_{2}}^{\left(k_{2}\right)}+\bar{J}_{\ell_{1}}^{\left(k_{1}\right)} \bar{J}_{\ell_{2}}^{\left(k_{2}\right) *}+\right. \\
& -2 e^{-i\left(\mu_{H}+\mu v\right)} J_{\ell_{1}}^{\left(k_{1}\right)} \bar{J}_{\ell_{2}}^{\left(k_{2}\right)}+ \\
& -K_{\ell_{1}}^{\left(k_{1}\right)} K_{\ell_{2}}^{\left(k_{2}\right) *}-{\overline{K_{\ell}}}_{\ell_{1}}^{\left(k_{1}\right)} \bar{K}_{\ell_{2}}^{\left(k_{2}\right) *}+ \\
& \left.+2 e^{-i\left(\mu_{H}-\mu_{v}\right)} K_{\ell_{1}}^{\left(k_{1}\right)} \bar{K}_{\ell_{2}}^{\left(k_{2}\right) *}\right\}
\end{aligned}
$$


where $\mathrm{J}_{\ell}^{(\mathrm{k}) *}$ and $\mathrm{K}_{\ell}^{(\mathrm{k}) *}$ are the complex conjugates of the rotations $\mathrm{J}_{\ell}^{(\mathrm{k})}$ and $\mathrm{K}_{\ell}^{(\mathrm{k})}$ and $\overline{\mathrm{J}}_{\ell}^{(\mathrm{k})}$, and $\overline{\mathrm{K}}_{\ell}^{(\mathrm{k})}$ are obtained from $\mathrm{J}_{\ell}^{(\mathrm{k})}$ and $\mathrm{K}_{\ell}^{(\mathrm{k})}$ by inverting the sign to the phase differences $\delta$ appearing in the phase factors of Eqs. (55 and 56). In particular, with a convenient expansion, with respect to the order of magnitude of the errors,

$$
\begin{aligned}
& \operatorname{Det}(n+\bar{m})= \\
& \begin{array}{r}
=\left|J_{-}\right|^{2} \sin ^{2} \pi\left(\nu_{H}+\nu_{V}\right)-\left|J_{+}\right|^{2} \sin ^{2} \pi\left(\nu_{H}-\nu_{V}\right)+ \\
-\frac{1}{16} \sum_{\ell=1}^{4} \operatorname{Real}\left\{J_{-}\left[J_{\ell}^{(3)}+\bar{J}_{\ell}^{(3)}\right]+J_{-}^{*}\left[J_{\ell}^{(3)}+\bar{J}_{\ell}^{(3) *}\right]+\right. \\
\left.\quad-2\left(J_{-} \bar{J}_{\ell}^{(3)}+J_{-}^{*} J_{\ell}^{(3)}\right) e^{-i\left(\mu_{H}+\mu_{V}\right)}\right\}+ \\
+\frac{1}{16} \sum_{\ell=1}^{4} \operatorname{Real}\left\{J_{+}\left[K_{\ell}^{(3)}+\bar{K}_{\ell}^{(3)}\right]^{*}+J_{+}^{*}\left[K_{\ell}^{(3)}+\bar{K}_{\ell}^{(3)}\right]+\right. \\
\left.\quad-2\left(J_{+} \bar{K}_{\ell}^{(3) *}+J_{+}^{*} K_{\ell}^{(3)}\right) e^{-i\left(\mu_{H}-\mu_{V}\right)}\right\}+
\end{array}
\end{aligned}
$$

+6 th and higher order terms.

At the same time

$$
\begin{aligned}
& \operatorname{Tr} A=2 \cos \mu_{H}+\sum_{\substack{k=2 \\
\text { ve: }}}^{N} \operatorname{Tr} A^{(k)} \\
& \operatorname{Tr} B=2 \cos \mu_{V}+\sum_{\substack{k=2 \\
\text { even }}}^{N} \operatorname{Tr} B^{(k)}
\end{aligned}
$$

But, with $k$ an even integer,

$$
\begin{aligned}
& A^{(k)}=\left(\frac{i}{2}\right)^{k} \sum_{\ell=1}^{h_{k}}\left[J_{\ell}^{(k)}+K_{\ell}^{(k)} C\right] e^{-i \mu_{H}} \\
& B^{(k)}=\left(\frac{i}{2}\right)^{k} \sum_{\ell=1}^{h_{k}}\left[\bar{J}_{\ell}^{(k)}+\bar{K}_{\ell}^{(k)} C\right] e^{-i \mu_{V}}
\end{aligned}
$$

Then it is easily seen that (see Appendix A)

$$
\begin{aligned}
& \operatorname{Tr} A^{(k)}=2\left(\frac{i}{2}\right)^{k} \operatorname{Real}\left[e^{-i \mu_{H}} \sum_{\ell} J_{\ell}^{(k)}\right] \\
& \operatorname{Tr} B^{(k)}=2\left(\frac{i}{2}\right)^{k} \operatorname{Real}\left[e^{-i \mu_{\nu}} \sum_{\ell} \bar{J}_{\ell}^{(k)}\right]
\end{aligned}
$$


where the driving terms of the sum mode do not enter at all. In particular,

$$
\begin{aligned}
\operatorname{Tr} A^{(2)}-\operatorname{Tr} B^{(2)} & = \\
& =\left(\operatorname{Real} J_{2}^{(2)}\right) \sin \pi\left(\nu_{H}+\nu_{V}\right) \sin \pi\left(\nu_{H}-\nu_{V}\right)+ \\
& -\left(\operatorname{Imag} J_{2}^{(2)}\right) \cos \pi\left(\nu_{H}+\nu_{V}\right) \sin \pi\left(\nu_{H}-\nu_{V}\right)+ \\
& +\left(\operatorname{Real} J_{1}^{(2)}\right) \sin \pi\left(\nu_{H}+\nu_{V}\right) \sin \pi\left(\nu_{H}-\nu_{V}\right)+ \\
& -\left(\operatorname{Imag} J_{1}^{(2)}\right) \sin \pi\left(\nu_{H}+\nu_{V}\right) \cos \pi\left(\nu_{H}-\nu_{V}\right) .
\end{aligned}
$$

We have thus derived explicit expressions for $\cos \mu_{1}$ and $\cos \mu_{2}$ which are valid to any order and that are given by the combination of Eqs. (82), (84-85) and (88-89) inserted in Eqs. (79-81).

\subsection{Stability Conditions}

The motion of a particle in the presence of skew quadrupole errors is stable only when each of the four eigenvalues has an absolute value which in magnitude is less than unit. This requires the simultaneous satisfaction of two distinct stability conditions; namely

$$
(\operatorname{Tr} A-\operatorname{Tr} B)^{2}+4 \operatorname{Det}(n+\bar{m}) \geq 0
$$

and, when this is satisfied,

$$
\left|\cos \mu_{1,2}\right| \leq 1
$$

where $\cos \mu_{1,2}$ is given by Eq. (81). In the absence of the skew quadrupole errors, $\mu_{1}$ and $\mu_{2}$ reduce to $\mu_{\mathrm{H}}$ and $\mu_{\mathrm{V}}$. In this case, the first condition is always satisfied and we shall assume that also the second stability condition is fulfilled, so that $\mu_{\mathrm{H}}$ and $\mu_{\mathrm{V}}$ are real quantities.

There are two cases of interest:

(i) Equal Tunes, where the vertical and horizontal betatron tunes $\nu_{\mathrm{V}}$ and $\nu_{\mathrm{H}}$ have the same fractional part, that is

$$
\nu_{\mathrm{H}}-\nu_{\mathrm{V}}=\mathrm{m}
$$

with m any integer or zero.

(ii) Opposite Tunes, where

$$
\nu_{\mathrm{H}}+\nu_{\mathrm{V}}=\mathrm{m}
$$

Both of these cases correspond to $\operatorname{Tr} A^{(0)}=\operatorname{Tr} B^{(0)}$. Then $(\operatorname{Tr} A-\operatorname{Tr} B)^{2}$ is of course always a positive quantity but small, of at least fourth- order in the strength parameter of the errors.

It is seen from Eq. (83) that, to third-order terms included, Det $(n+\bar{m})$ is positive in the first case and thus the first stability condition Eq. (91) is always satisfied. In the case of opposite tunes, Det $(n+\bar{m})$ is a negative quantity and thus Eq. (91) is not necessarily satisfied; in this case the motion is unstable. It is then preferable to design and operate an accelerator with the case shown by Eq. (93). The magnitude of the integer $\mathrm{m}$ is here not important. In the following we shall consider only the case of equal tunes. 


\subsection{Tune-Splitting}

This is defined as half of the diffcrence between the shifted tunes, namely

$$
\Delta=\frac{\mu_{2}-\mu_{1}}{4 \pi}
$$

It is important that this quantity is made as small as possible. From Eqs. (79-81) we obtain

$$
\Delta \approx \frac{1}{4 \pi \sin \mu_{0}} \sqrt{\frac{1}{4}(\operatorname{Tr} \mathrm{A}-\operatorname{Tr} \mathrm{B})^{2}+\operatorname{Det}(\mathrm{n}+\overline{\mathrm{m}})}
$$

where $\mu_{0}$ is the average of $\mu_{1}$ and $\mu_{2}$. We have

$$
\operatorname{Tr} A^{(0)}-\operatorname{Tr} B^{(0)}=0
$$

and from Eq. (90)

$$
\operatorname{Tr} A^{(2)}-\operatorname{Tr} B^{(2)}=-\left(\operatorname{Imag} J_{1}^{(2)}\right) \sin \mu_{0}
$$

so that (TrA - TrB $)^{2}$ in the square root of Eq. (96) is a small quantity of at least fourth-order in the magnitude of the errors strength parameter. On the other side, to the lowest order

$$
\operatorname{Det}(\mathrm{n}+\overline{\mathrm{m}})=|\mathrm{J}-|^{2} \sin ^{2} \mu_{0}
$$

Thus since we expect that $\left|J_{1}^{(2)}\right|$ to be considerably smaller than $\left|J_{-}\right|$, the tune-splitting for an uncorrected machine is

$$
|\Delta| \approx \frac{1}{4 \pi}\left|J_{-}\right|
$$

which, as one would have expected is of first order in the strength parameters.

\subsection{Correction Strategy}

Suppose that the goal is to correct for an unreasonably large tune-splitting. Then from Eq. (100) it is obvious that one wants to place two families of correctors around the ring to compensate for the $\mathrm{J}_{-}$driving term, which is given by $\mathrm{Eq}$. (59). In principle one will require also the correction of the $\mathrm{J}_{+}$driving term, given by $\mathrm{Eq} .(60)$, since because of the regular gradient errors, not necessarily the two tunes satisfy Eq. (93) exactly. The correction of the $\mathrm{J}_{+}$ driving term can be accomplished with the addition of two more families of skew quadrupole correctors. It is desirable that the two sets of correctors are normal to each other so that the two driving terms, $\mathrm{J}_{-}$and $\mathrm{J}_{+}$, can be adjusted independently.

Inspection of Eq. (83) shows that, setting $\mathrm{J}_{-}=\mathrm{J}_{+}=0$, corrects also the fourth- order terms of $\operatorname{Det}(n+\bar{m})$ automatically. Thus, after correction, $\operatorname{Det}(n+\bar{m})$ is a small quantity of at least 6-th order in the error strength parameters. On the other side this method of correction would still leave (TrA - TrB) ${ }^{2}$ to at least fourth-order; so that now the first term under the square root of $\mathrm{Eq}$. (96) dominates with respect to the second term and

$$
\Delta \approx \frac{\operatorname{Tr} A-\operatorname{Tr} B}{16 \pi \sin \mu_{0}}
$$

which according to Eq. (90) shows a secont-order dependence with the magnitude of the errors. 
In some cases, even this residual tune-splitting may be too large to be tolerateci. Then a further correction to higher order is required. In the case of equal tunes, the lowest order contribution to Eq. (101) is given by the last term at the right hand side of Eq. (90),

$$
\Delta \approx \frac{\operatorname{Imag}\left[\mathrm{J}_{1}^{(2)}\right]}{16 \pi}
$$

In principle two more families of correctors are required for the control of the driving term $\mathrm{J}_{1}^{(2)}$, which together to those for $\mathrm{J}_{+}$and $\mathrm{J}_{-}$add to a total of six families, divided in three groups. It is to be observed that the driving term $\mathrm{J}_{1}^{(2)}$ is actually a second-order corrilation factor among all the errors and correctors and thus of difficult control. It would be important that the correctors set for $\mathrm{J}_{+}$and $\mathrm{J}_{-}$adjustment are placed in such a way that do not affect the overall $\mathrm{J}_{1}^{(2)}$ driving term. Similarly it would be desirable that the correctors for the control of $\mathrm{J}_{1}^{(2)}$ do not change $\mathrm{J}_{+}$and $\mathrm{J}_{-}$.

\subsection{References}

1. E.D. Courant and H.S. Snyder, Ann. Phys. 3, 1 (1958).

2. D.A. Edwards and L.C. Teng, IEEE Trans. Nucl. Sci. Vol. NS-20, No. 3, p. 885 (1973).

3. F. Willeke and G. Ripken, "Methods of Beam Optics", AIP Conference Proceedings 184 , Vol. 1, p. 758.

\section{Appendix A}

Let $\mathrm{R}$ represent a $2 \times 2$ matrix which performs a rotation in the anticlockwise direction by an angle $\theta$ of a two-dimensional vector in a plane,

$$
R=\left(\begin{array}{rr}
\cos \theta & -\sin \theta \\
\sin \theta & \cos \theta
\end{array}\right)
$$

which in complex number notation can be also represented by the operator

$$
R=e^{i \theta}
$$

Let $\mathrm{n}$ and $\mathrm{m}$ be two independent linear combinations of a finite number of rotations, that is

$$
\begin{aligned}
& n=\sum_{s} a_{s} R_{n} . \\
& m=\sum_{r} b_{r} R_{m} .
\end{aligned}
$$

where the coefficients $a_{s}$ and $b_{r}$ are real numbers.

If $R_{n_{1}}$ rotates the vector by an angle $\theta_{n_{0}}$ and $R_{m}$, by an angle $\theta_{m_{r}}$, then the product $R_{n_{2}}$ $R_{m_{r}}$ is also a rotation matrix-operator of the form given by Eq. (A.1) with the angle $\theta_{n_{0}}+\theta_{m_{r}}$.

Let $R^{*}$ denote the complex conjugate of $R$ given by Eq. (A.2), that is

$$
\mathrm{R}^{*}=\mathrm{e}^{-\mathrm{i} \theta}
$$


This operator rotates the vector by an angle $\theta$ but in the clockwise direction, so that

$$
R R^{*}=R^{*} R=1
$$

In matrix notation, it is seen from Eq. (A.1), that

$$
\mathrm{R}^{*}=\mathrm{R}^{-1}
$$

where $\mathrm{R}^{-1}$ is the inverse of the $2 \times 2$ matrix.

It is easily seen that

$$
\operatorname{Det}(R)=1
$$

and

$$
\begin{aligned}
\operatorname{Tr}(R) & =2 \cos \theta \\
& =2 \operatorname{Real}(R)
\end{aligned}
$$

Also

$$
\begin{aligned}
\operatorname{Det}(n) & =\operatorname{Real}\left(n n^{*}\right) \\
& =\sum_{s} \sum_{r} a_{s} a_{r} \cos \left(\theta_{n,}-\theta_{m_{r}}\right) \\
& =\operatorname{Real}\left(\sum_{s} \sum_{r} a_{s} a_{r} R_{n}, R_{m_{r}}^{*}\right)
\end{aligned}
$$

and

$$
\begin{aligned}
\operatorname{Tr}(n) & =2 \sum_{s} a_{s} \cos \theta_{n}, \\
& =2 \operatorname{Real} \sum_{s} a_{s} R_{n} .
\end{aligned}
$$

If $\mathrm{C}$ is the complex conjugate operator which in matrix notation is defined by

$$
C=\left(\begin{array}{rr}
1 & 0 \\
0 & -1
\end{array}\right)
$$

then

$$
\operatorname{Tr}(\mathrm{nC})=0
$$

and

$$
\operatorname{Det}(\mathrm{nC})=-\operatorname{Det}(\mathrm{n})
$$

Since the product $\mathrm{p}=\mathrm{n} \cdot \mathrm{m}$ is also a linear combination of rotations with real coefficients, then all equations above hold also for $\mathrm{p}$.

\section{Appendix B}

To obtain explicit expressions of the eigenvalues we need to calculate Det $(n+\bar{m})$. We note first of all that

$$
\operatorname{Det}(n+\bar{m})=\operatorname{Det}(n)+\operatorname{Det}(m)+\operatorname{Tr}(n m)
$$


We can write concisely

$$
\begin{aligned}
& n=n^{-}+n^{+} C \\
& m=m^{-}+m^{+} C
\end{aligned}
$$

where the first terms, shown with the sign minus, are the contribution from the difference mode, and the second terms denote the contribution from the sum mode. Taking into account Eqs. (A.13-14) and Eq. (B.1) we have

$$
\begin{aligned}
\operatorname{Det}(n) & =\operatorname{Det}\left(n^{-}\right)+\operatorname{Det}\left(n^{+} C\right)+\operatorname{Tr}\left(\overline{n^{-}} n^{+} C\right) \\
& =\operatorname{Det}\left(n^{-}\right)-\operatorname{Det}\left(n^{+}\right)
\end{aligned}
$$

Similarly

$$
\operatorname{Det}(\mathrm{m})=\operatorname{Det}\left(\mathrm{m}^{-}\right)-\operatorname{Det}\left(\mathrm{m}^{+}\right)
$$

At the same time

$$
\begin{aligned}
\operatorname{Tr}(n m) & =\operatorname{Tr}\left(n^{-} m^{-}\right)+\operatorname{Tr}\left(n^{+} m^{+^{-}} C\right)+ \\
& +\operatorname{Tr}\left(n^{-} m^{+} C\right)+\operatorname{Tr}\left(n^{+} m^{-}{ }^{-} C\right) \\
& =\operatorname{Tr}\left(n^{-} m^{-}\right)+\operatorname{Tr}\left(n^{+} m^{+^{-}}\right)
\end{aligned}
$$

So that, finally,

$$
\begin{aligned}
\operatorname{Det}(n+\bar{m}) & =\operatorname{Det}\left(n^{-}\right)-\operatorname{Det}\left(n^{+}\right)+ \\
& +\operatorname{Det}\left(m^{-}\right)-\operatorname{Det}\left(m^{+}\right)+ \\
& +\operatorname{Tr}\left(n^{-} m^{-}\right)+\operatorname{Tr}\left(n^{+} m^{+^{+}}\right)
\end{aligned}
$$

Taking into account Eqs. (A.10-11) we then obtain Eq. (82).

\section{DISCLAIMER}

This report was prepared as an account of work sponsored by an agency of the United States Government. Neither the United States Government nor any agency thereof, nor any of their employees, makes any warranty, express or implied, or assumes any legal liability or responsibility for the accuracy, completeness, or usefulness of any information, apparaius, product, or process disclosed, or represents that its use would not infringe privately owned rights. Reference herein to any specific commercial product, process, or service by trade name, trademark, manufacturer, or otherwise does not necessarily constitute or imply its endorsement, recommendation, or favoring by the United States Government or any agency thereof. The views and opinions of authors expressed herein do not necessarily state or reflect those of the United States Government or any agency thereof. 

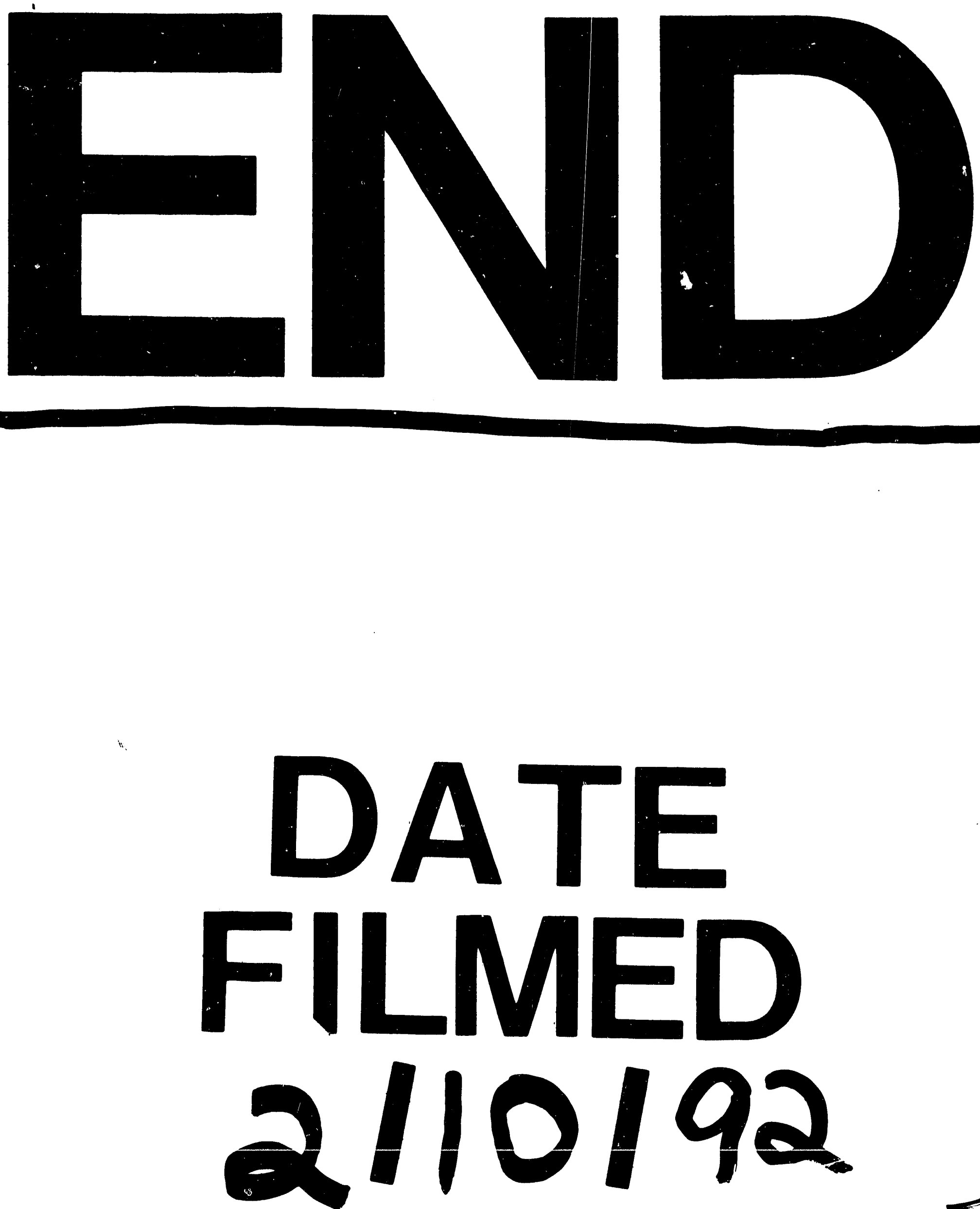

$I$ 
